\title{
The Incredible Lightness of Water Vapor
}

\author{
DA YANG AND SETH D. SEIDEL \\ University of California, Davis, and Lawrence Berkeley National Laboratory, Berkeley, California
}

(Manuscript received 4 April 2019, in final form 12 November 2019)

\begin{abstract}
The molar mass of water vapor is much less than that of dry air. This makes a moist parcel lighter than a dry parcel of the same temperature and pressure. This effect is referred to as the vapor buoyancy effect and has often been overlooked in climate studies. We propose that the vapor buoyancy effect increases Earth's outgoing longwave radiation (OLR) and that this negative radiative effect increases with warming, stabilizing Earth's climate. We illustrate this mechanism in an idealized tropical atmosphere, where there is no horizontal buoyancy gradient in the free troposphere. Temperature increases toward dry atmosphere columns to compensate the reduced vapor buoyancy, increasing OLR by $O\left(1 \mathrm{~W} \mathrm{~m}^{-2}\right)$ at the reference climate. In warmer climates, the temperature difference between moist and dry columns would increase as a result of increasing atmospheric water vapor, leading to enhanced radiative effect and thereby stabilizing Earth's climate. We estimate that this feedback strength is about $O\left(0.2 \mathrm{~W} \mathrm{~m}^{-2} \mathrm{~K}^{-1}\right)$ in the idealized atmosphere, which compares to cloud feedback and surface albedo feedback in the current climate. We further show evidence from observations and real-gas radiative transfer calculations for a significant radiative effect of vapor buoyancy in the tropical atmosphere.
\end{abstract}

\section{Introduction}

How fast would Earth's climate respond to increasing $\mathrm{CO}_{2}$ (Manabe and Wetherald 1975; Flato et al. 2013; Collins et al. 2013)? Why is tropical climate more stable than extratropical climate (Holland and Bitz 2003; Polyakov et al. 2002; Pierrehumbert 1995)? What sets the inner edge of the habitable zone of Earth-like planets (Yang and Abbot 2014; Pierrehumbert 2010a)? Understanding and accurately estimating climate feedbacks are key to address these pressing questions.

The importance of water vapor seems to be widely recognized in the literature of climate feedbacks (Manabe and Wetherald 1967; Ingersoll 1969; Held and Soden 2000; Flato et al. 2013). Previous studies have focused on three basic effects of water vapor: E1) water vapor is a greenhouse gas; E2) water vapor can condense to liquid water and release latent heat; and E3) saturation vapor pressure increases with temperature exponentially. The combination of E1 and E3 gives rise to the water vapor feedback, the dominant positive climate feedback (Manabe and Wetherald 1967; Held and Soden 2000; Flato et al. 2013). Increasing temperature leads to more water vapor, which leads to an enhanced greenhouse effect, warming the planet further. The water vapor

Corresponding author: Da Yang, dayang@ucdavis.edu feedback could even lead to a runaway greenhouse state when the atmosphere is sufficiently opaque to longwave radiation that the outgoing longwave radiation (OLR) is insensitive to surface temperature (Ingersoll 1969). The combination of E2 and E3 gives rise to the (tropical) lapse rate feedback, a negative climate feedback in the tropical atmosphere (Flato et al. 2013). Increasing temperature leads to more water vapor, which leads to a weaker lapse rate in the tropical atmosphere. This effect increases upper troposphere temperature more than in the lower troposphere, leading to higher emission of OLR, which cools the planet. At higher latitudes, the temperature lapse rate is no longer controlled by moist convection, so the lapse rate feedback is less constrained. Both feedbacks are among the five most important climate feedbacks in the Intergovernmental Panel on Climate Change (IPCC) reports and have been extensively evaluated in general circulation models (GCMs) (Flato et al. 2013).

However, the lightness of water vapor has been completely overlooked in the context of climate feedbacks. In Earth's atmosphere, buoyancy is represented by the virtual temperature [e.g., see Eq. (5) in Yang (2018a)]. We define the virtual temperature

$$
T_{v}=T\left(\frac{1+r / \varepsilon}{1+r}\right)
$$


where $T$ is temperature, $r$ is water vapor mixing ratio, and $\varepsilon=M_{v} / M_{d}$. The molar mass of water vapor $M_{v}$ is $18 \mathrm{~g} \mathrm{~mol}^{-1}$, significantly lighter than that of dry air $M_{d}$, which is $29 \mathrm{~g} \mathrm{~mol}^{-1}$. This makes a moist parcel lighter than a dry parcel of the same temperature and pressure (Emanuel 1994). Here we refer to this as the vapor buoyancy effect, although it is also referred to as the virtual effect (Yang 2018a,b). The vapor buoyancy effect makes $T_{v}$ slightly higher than $T$ by $O(1 \mathrm{~K})$ in the tropical atmosphere, which is of the same order of magnitude as typical temperature fluctuations there.

We propose that the vapor buoyancy effect can increase Earth's OLR and help to stabilize Earth's climate by regulating the atmosphere's thermal structure. Figure 1 shows the temperature and virtual temperature (buoyancy) fields in the moisture space from $2^{\circ} \mathrm{S}$ to $2^{\circ} \mathrm{N}$ using NASA AIRS data. In the free troposphere $(p<850 \mathrm{hPa})$, buoyancy is horizontally uniform because of the small Coriolis parameter and efficient gravity waves (Charney 1963; Bretherton and Smolarkiewicz 1989; Sobel et al. 2001; Yang 2018a). However, temperature increases toward the dry columns as a result of the vapor buoyancy effect. There, moisture and its associated vapor buoyancy are reduced. To maintain uniform buoyancy, temperature has to increase. We propose that the temperature tilt would increase with climate warming due to increasing atmospheric moisture, leading to enhanced OLR over the dry area. This is a negative feedback that can help to stabilize Earth's climate.

Previous studies implied that vapor buoyancy could make temperature increase toward the dry columns in the tropical atmosphere (Tompkins 2001; Bretherton and Smolarkiewicz 1989; Bretherton et al. 2005; Yang 2018a,b). However, they have often considered this effect to be small and negligible, simplifying the dynamics according to a weak temperature gradient approximation (Sobel et al. 2001). These studies, therefore, did not consider that its radiative effect is significant, which is the novelty of this study.

In section 2, we explain our hypothesis in detail. We first illustrate how the vapor buoyancy effect increases Earth's OLR (a negative radiative effect) and then explain why this effect strengthens with climate warming. In section 3, we derive a simple model for the radiative effect and feedback strength of the vapor buoyancy effect. We then use the simple model to make order-ofmagnitude estimates for the radiative effect and feedback strength. In section 4 , we estimate the radiative effect by using tropical sounding profiles with a real-gas radiative transfer model. In section 5, we conclude and discuss implications for the climate stability of Earth and other planets.

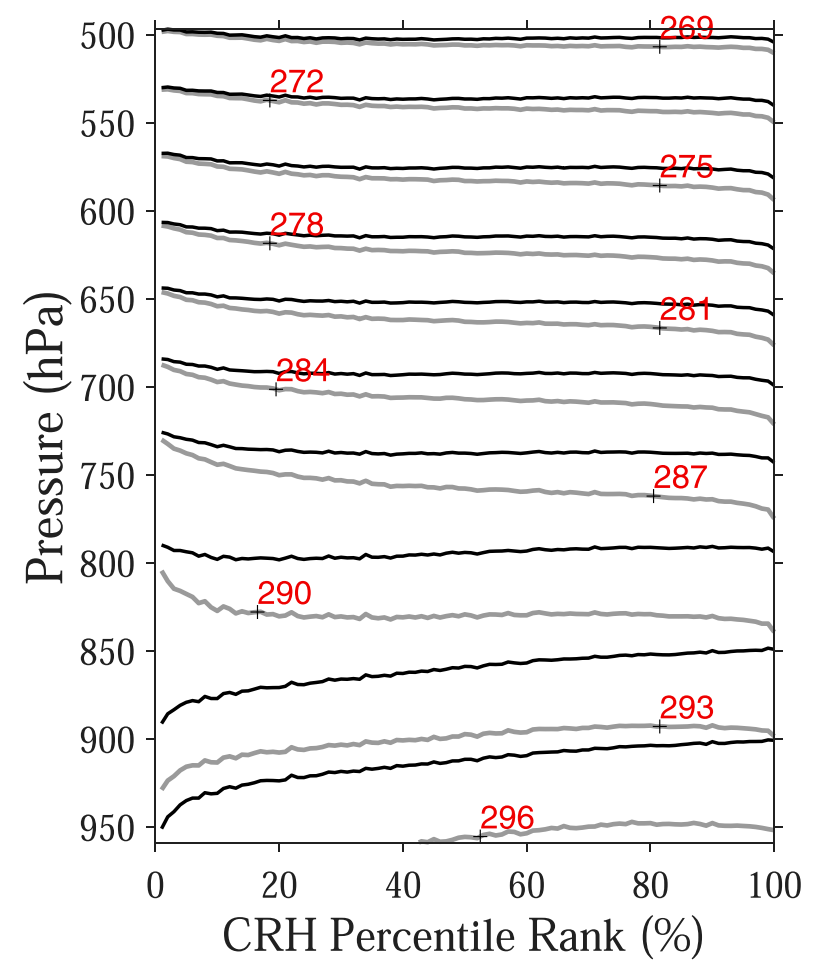

FIG. 1. Temperature and virtual temperature fields in moisture space using NASA AIRS data from $2^{\circ} \mathrm{S}$ to $2^{\circ} \mathrm{N}$ for the calendar year 2017. Black contours correspond to virtual temperature, and gray contours correspond to temperature. The $x$ axis is column relative humidity $(\mathrm{CRH})$ rank, where $\mathrm{CRH}$ was calculated as precipitable water divided by saturation precipitable water above the altitude of $850 \mathrm{hPa}$. The driest columns are to the left, and the moistest columns are to the right. The temperature contours deviate from virtual temperature contours as a result of vapor buoyancy. In the free troposphere, the horizontal buoyancy gradient is weak. Therefore, virtual temperature contours are approximately flat and temperature increases toward dry columns. In the planetary boundary layer, surface drag can sustain significant pressure gradients, and thereby buoyancy gradients. There, both virtual temperature and temperature increase toward the moist columns, forming a low pressure center over the upwelling branch of the atmospheric circulation.

\section{Hypothesis}

We propose that vapor buoyancy can increase OLR (a negative radiative effect) due to a clear-sky effect, and that the radiative effect increases with climate warming. Figure 2 illustrates our hypothesis by comparing OLR from two stand-alone atmospheres with overturning circulations: one considers the vapor buoyancy effect (control) and the other does not consider this effect. The overturning circulation is analogous to the Walker circulation or convective self-aggregation in the tropics (Bretherton et al. 2005; Pritchard and Yang 2016; Yang and Ingersoll 2013, 2014; Yang 2019). The upwelling branch of the circulation is associated with deep 

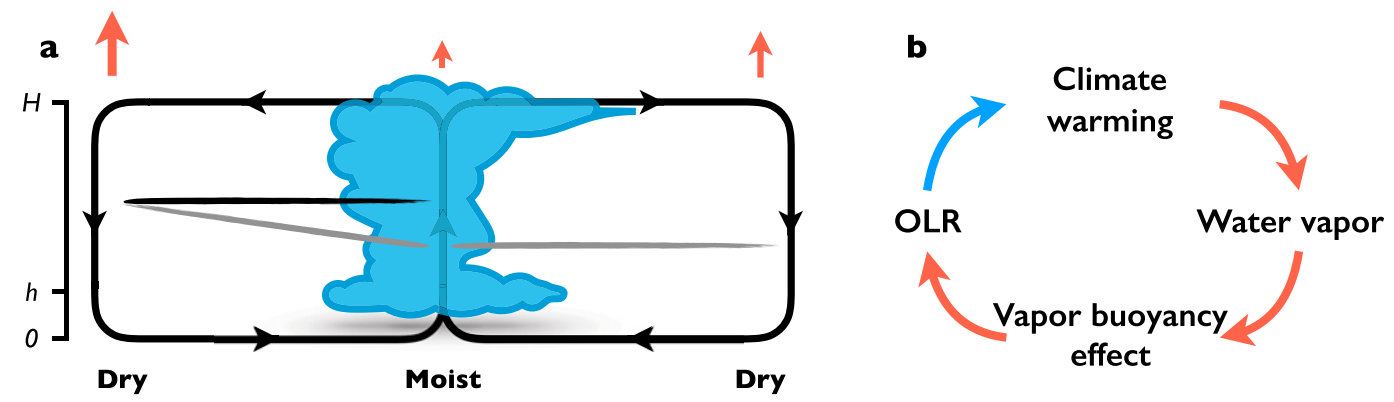

FIG. 2. Schematic diagrams: (a) The vapor buoyancy effect increases OLR in the tropical atmosphere. This figure depicts two stand-alone atmospheres: the control atmosphere (on the left) and the no-vapor-buoyancy atmosphere (on the right). The horizontal axis is $x$ or $\mathrm{CRH}$; the vertical axis is height ( $h=$ boundary layer height; $H=$ tropopause height). The gray lines represent temperature contours, and the black line represents a buoyancy or virtual temperature contour. The orange arrows represent OLR emission: large or small arrows correspond to more or less OLR, respectively. (b) The negative climate feedback. Orange arrows represent an increase effect; the blue arrow represents a decrease effect.

convection and moist air, and the downwelling branch is associated with clear skies and dry air. For illustrative purposes, we make a few simplifications: S1) the two atmospheres are nonrotating, S2) the two atmospheres sit above ocean surface with the same, uniform surface temperature, and S3) the two atmospheres have the same water vapor distribution. The first two simplifications are relevant to the tropical atmosphere as the rotation effect and surface temperature gradient are both weak in the tropics. The third simplification is often required when calculating a radiative effect.

Figure 2 shows that the control atmosphere emits more OLR than the no-vapor-buoyancy atmosphere due to the higher temperature in the dry area. OLR is primarily a function of temperature and water vapor mixing ratio $r$. When $r$ remains the same in the two atmospheres (S3), the OLR difference would come from temperature differences between the two atmospheres. Here we provide physical intuition on why there should be temperature differences, leaving a detailed derivation for section $3 \mathrm{e}$. The temperature profiles of moist areas in the two atmospheres are set by convective plumes. Because these convective plumes rise from the same surface temperature, the temperature profiles should be almost identical in the two moist areas. Temperature profiles in the dry areas, however, differ significantly, leading to differences in OLR. According to long-accepted results in geophysical fluid dynamics, the horizontal buoyancy gradient is negligible in the free troposphere without rotation because gravity waves can effectively smooth out buoyancy anomalies (Charney 1963; Sobel et al. 2001). We refer to this effect as the weak buoyancy gradient (WBG) approximation (Yang 2018a). In the control atmosphere, buoyancy is a function of both temperature and and water vapor mixing ratio $r$ due to the vapor buoyancy effect. The horizontal moisture gradient then leads to a horizontal temperature gradient: dry air is warmer than moist air. In the no-vapor-buoyancy atmosphere, the free-troposphere temperature is horizontally invariant, as buoyancy is a function of temperature only. The dry column of the control atmosphere, therefore, is warmer than that of the no-vapor-buoyancy atmosphere by $O(1 \mathrm{~K})$, leading to enhanced OLR. The spectrum of $\mathrm{H}_{2} \mathrm{O}$ in the longwave is also sensitive to temperature. However, this impact is likely small.

In warmer climates, the vapor buoyancy effect would become more significant due to increasing water vapor. Therefore, we expect that the radiative effect due to the vapor buoyancy also increases with climate warming. This is a negative climate feedback (Fig. 2b). The proposed mechanism relies on ample atmospheric water vapor, so it would be most effective in stabilizing the tropical climate. In principle, this feedback should have been represented by climate models. However, it has not been evaluated or even discussed.

We will construct a simple model of the proposed feedback mechanism. This will give an order-of-magnitude estimate of the associated radiative effect and the rate at which it increases with climate warming.

\section{A simple model}

We construct a simple model based on the schematic diagram (Fig. 2). Each atmosphere with overturning circulations is represented by a dry column and a moist column (Pierrehumbert 1995). Because the the two atmospheres' respective moist columns would have the same temperature profiles, the OLR difference primarily comes from their dry columns, which we will focus on. Again, we aim to estimate the "radiative effect" due to the vapor buoyancy effect. Therefore, we 
assume that all basic dynamic (e.g., circulation and pressure) and thermodynamic features (e.g., moisture) are the same in the two atmospheres-one with the vapor buoyancy effect, and the other without it.

The goal of this simple model is to provide an order-ofmagnitude understanding of our hypothesis. Therefore, we employ a two-band radiative transfer model. The twoband model is more realistic than a gray atmosphere model by allowing two absorption bands with distinct absorption coefficients, leading to different emission levels. The two-band model is, on the other hand, much simpler than a real-gas radiative transfer model, so the results are easier to interpret. To further test our hypothesis and the validity of the simple model, we also show calculation results using a real-gas radiative transfer model in the appendix. The results are in good agreement between the two models, suggesting that our hypothesis is based on robust physics and not on model details.

\section{a. The two-band model}

We consider a plane-parallel atmosphere and use the two-stream approximation in the following calculations. Only clear-sky longwave (IR) radiation is considered, and the IR opacity is mainly due to water vapor. Here we parameterize the water vapor absorption spectrum by two broad bands that occupy roughly equal fractions of blackbody emission at Earth-like temperatures (Beucler and Cronin 2016): one with a strong absorption coefficient $\kappa_{S}$ and the other with a weak absorption coefficient $\kappa_{W}$.

We first consider one absorption band with any given $\kappa$. OLR is defined as

$$
\mathrm{OLR}^{\kappa} \equiv F^{\uparrow}(p=0)-F^{\downarrow}(p=0),
$$

where $F^{\uparrow}$ and $F^{\downarrow}$ are upward and downward longwave radiative fluxes (Pierrehumbert 2010b). We know that $F^{\downarrow}(0) \approx 0$, so we shall solve for $F^{\uparrow}(0)$ in the gray atmosphere, which is given by

$$
d F^{\uparrow} / d \tau=F^{\uparrow}-\sigma T^{4},
$$

where $T$ is temperature, $\tau$ is optical depth, and $\sigma$ is the Stefan-Boltzmann constant. This is the two-stream Schwarzschild equation (Pierrehumbert 2010b). We integrate Eq. (3) and get

$$
F^{\uparrow}(0)=e^{-\tau_{s}} F^{\uparrow}\left(\tau_{s}\right)+\int_{0}^{\tau_{s}} \sigma T^{4} e^{-\tau^{\prime}} d \tau^{\prime} .
$$

The OLR is then given by

$$
\mathrm{OLR}^{\kappa}=e^{-\tau_{s}} \sigma T_{s}^{4}+\int_{0}^{\tau_{s}} \sigma T^{4} e^{-\tau^{\prime}} d \tau^{\prime}
$$

where $A_{s}$ represents the surface value of some variable $A$, and we have used $F^{\uparrow}\left(\tau_{s}\right)=\sigma T_{s}^{4}$. This equation shows that OLR has two components: one is the surface contribution, and the other is the atmosphere contribution.

We now use Eq. (5) to calculate the OLR difference between the two atmospheres, each containing one moist and one dry column. We remind the readers that $T_{s}, r$, and thereby $\tau$ of the two atmospheres are identical, so the OLR difference primarily comes from dry columns, between which there is significant difference in air temperature. The OLR difference of the dry column is given by

$$
\begin{aligned}
\Delta \mathrm{OLR}^{\kappa} & \equiv \mathrm{OLR}_{v}^{\kappa}-\mathrm{OLR}_{\mathrm{nv}}^{\kappa} \approx \int_{0}^{\tau_{s}} 4 \sigma T_{d}^{3} \Delta T e^{-\tau^{\prime}} d \tau^{\prime} \\
& \approx \int_{0}^{\tau_{s}} 4 \sigma T_{m}^{3} \Delta T e^{-\tau^{\prime}} d \tau^{\prime},
\end{aligned}
$$

where $\mathrm{OLR}_{v}$ and $\mathrm{OLR}_{\mathrm{nv}}$ represent OLR in the atmosphere with and without the vapor buoyancy effect. In the last equality, we assumed that $\left(T_{d}-T_{m}\right) / T_{m} \ll 1$. Because the strong and weak absorption bands occupy equal portions of the spectrum, the total OLR difference is given by

$$
\Delta \mathrm{OLR}=0.5\left(\Delta \mathrm{OLR}^{\kappa_{S}}+\Delta \mathrm{OLR}^{\kappa_{W}}\right) .
$$

To compute $\Delta \mathrm{OLR}$, we need information on $T_{m}, \Delta T, \tau$, and thereby $r$, which is the mixing ratio of water vapor.

\section{b. Temperature}

In Earth's tropical atmosphere, temperature profiles can be approximated by power-law relations of pressure:

$$
T=T_{s}\left(p / p_{s}\right)^{R_{d} \Gamma_{M} / g},
$$

where $T_{s}$ is the surface temperature, $p_{s}$ is surface pressure, $R_{d}$ is the gas constant for dry air, $\Gamma_{M}$ is the moist adiabatic lapse rate, and $g$ is gravity acceleration. This has been referred to as the "all-troposphere model" by Pierrehumbert (2010b), as the lapse rate is entirely determined by moist convection. Equation (8) fits the observed temperature profiles in the tropical troposphere, but introduces significant biases in the stratosphere (Beucler and Cronin 2016). Earth's OLR is dominated by tropospheric contributions, which justifies the use of Eq. (8).

\section{c. Moisture}

The water vapor mixing ratio $r$ is the ratio of the mass of water vapor to the mass of dry air and is given by

$$
r=\mathrm{RH} \times r^{*}(T, p),
$$


where $\mathrm{RH}$ is the relative humidity, and $r^{*}$ is the saturation mixing ratio. For the moist column, we assume that $r_{m}=r_{m}^{*}$ (i.e., $\mathrm{RH}=1$ ) at all vertical levels; for the dry column, we have $r=\beta r_{m}^{*}$, where $0<\beta<1$. Here $\beta$ is a more convenient parameter than the relative humidity of dry columns $\left(\mathrm{RH}_{d}\right)$. This is because, at given $T_{s}$, the two atmospheres' dry columns are of different temperatures, so they would have different $\mathrm{RH}$ values corresponding to the same mixing ratio. In reality, $\beta$ could have complicated vertical structures, which requires multiple parameters to describe. For the purpose of illustrating the proposed mechanism with minimal parameters, we take $\beta$ as a constant at all vertical levels. This simplification may affect the results quantitatively but will not affect the results qualitatively.

\section{d. Optical depth}

Outgoing longwave radiation is observed from space, so it would be convenient to define the optical depth $\tau$ as an increasing function as pressure. We thus require that $\tau(p=0)=0$. We write the optical depth as

$$
d \tau=\kappa r \frac{d p}{g}
$$

The optical depth would have different values for the two absorption bands: $d \tau=\kappa^{s} r d p / g$ for the strong band, and $d \tau=\kappa^{w} r d p / g$ for the weak band. In this model, we ignore the pressure-broadening effect and treat the absorption coefficients as constant: $\kappa^{s}=1.66 \times 0.1$ $\left(\mathrm{m}^{2} \mathrm{~kg}^{-1}\right)$ and $\kappa^{w}=1.66 \times 0.02\left(\mathrm{~m}^{2} \mathrm{~kg}^{-1}\right)$, where the factor of 1.66 is referred to as the diffusivity factor (Pierrehumbert 2010b). Here the absorption coefficients are consistent with previous modeling studies of similiar complexity (Ingersoll 1969; Pierrehumbert 2010b; Beucler and Cronin 2016). We can integrate Eq. (10) to obtain the optical depth at an arbitrary pressure level:

$$
\tau(p)=\int_{0}^{p} \kappa r \frac{d p^{\prime}}{g} .
$$

At surface $p=p_{s}$, we then have

$$
\tau_{s} \equiv \tau\left(p_{s}\right)=\int_{0}^{p_{s}} \kappa r \frac{d p^{\prime}}{g} .
$$

Here $\tau_{s}$ measures the optical depth at the surface, which is total optical depth of the atmospheric column.

\section{e. The $W B G$ approximation and $\Delta T$}

Buoyancy is horizontally homogenized in the tropical free troposphere (Fig. 1). We refer to this constraint as the weak buoyancy gradient (WBG) approximation
(Yang 2018a). This is an improvement of the weak temperature gradient (WTG) approximation, which neglects the vapor buoyancy effect (Charney 1963; Sobel et al. 2001). While the dynamics of the atmosphere respond to buoyancy, radiation responds to absolute temperature. WBG together with the vapor buoyancy effect leads to a warmer lower troposphere in the dry column. This warming yields greater OLR. In a moist atmosphere, buoyancy is related to the virtual temperature, which is given by

$$
T_{v}=T\left(\frac{1+r / \varepsilon}{1+r}\right)
$$

where $\varepsilon=M_{v} / M_{d}$, with $M_{v}$ and $M_{d}$ representing the molar mass of water vapor and dry air, respectively. In the free troposphere, uniform buoyancy requires the virtual temperature to be uniform across the moist and dry area:

$$
T_{m}\left(\frac{1+r_{m} / \varepsilon}{1+r_{m}}\right)=T_{d}\left(\frac{1+r_{d} / \varepsilon}{1+r_{d}}\right)
$$

We substitute $T_{d}=T_{m}+\Delta T_{\mathrm{WBG}}$ into Eq. (13) and get

$$
\Delta T_{\mathrm{WBG}}=T_{m}\left(\frac{1+r_{m} / \varepsilon}{1+r_{m}}-\frac{1+r_{d} / \varepsilon}{1+r_{d}}\right)\left(\frac{1+r_{d}}{1+r_{d} / \varepsilon}\right) .
$$

Equation (14) is derived without approximations about the amount of water vapor and the amplitude of $\Delta T$. Although this form is very accurate, we would like to simplify it by assuming water vapor is a trace gas: $r \ll 1$. This is a good assumption for the current climate and may still be good until surface temperature reaches $320 \mathrm{~K}$, at which temperature $r^{*}\left(p_{s}\right)=73 \mathrm{~g} \mathrm{~kg}^{-1}$. With this approximation, we get

$$
\Delta T_{\mathrm{WBG}}=T_{m}\left(\frac{1}{\varepsilon}-1\right)\left(r_{m}-r_{d}\right) .
$$

This simplified equation clearly tells that $\Delta T$ depends on the contrast, not just absolute values, of mixing ratio and molar mass.

The above calculation is more accurate in the free troposphere, where gravity waves efficiently smooth out buoyancy anomalies. Although there are no such constraints in the boundary layer, we can assume that $\Delta T=0$ at the surface because of the uniform sea surface temperature (SST). Therefore, we require $\Delta T$ to be equal to $\Delta T_{\mathrm{WBG}}$ in the free troposphere but to smoothly decay to 0 at surface:

$$
\Delta T=\Delta T_{\mathrm{WBG}}\left[1-\left(\frac{p}{p_{s}}\right)^{n}\right]
$$



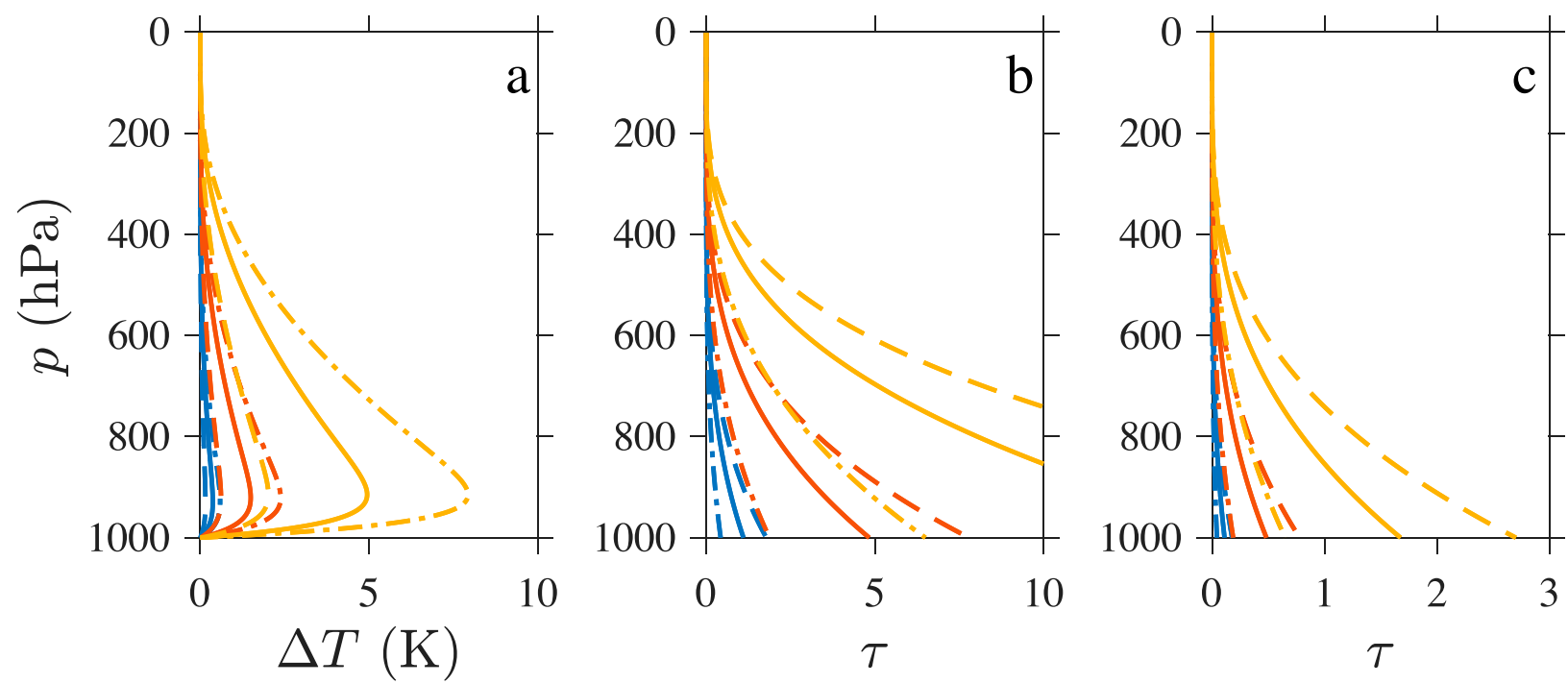

FIG. 3. Plots of (a) $\Delta T$ profiles (K), along with $\tau$ profiles for the (b) strong and (c) weak absorption bands. Blue is $280 \mathrm{~K}$, red is $300 \mathrm{~K}$, and yellow is $320 \mathrm{~K}$. Dot-dashed lines indicate $\beta=0.2$; solid lines indicate $\beta=0.5$, and dashed lines indicate $\beta=0.8$.

where $n$ controls the decay rate with pressure. The $p / p_{s}$ term would decay faster with large $n$ and slower with small $n$, so different $n$ could potentially result in different amplitudes and altitudes of the maximum temperature difference. However, we find that the values of $\triangle$ OLR and its sensitivity to surface temperature only change by $50 \%$ while we vary $n$ over an order of magnitude, from 5 to 50 . Therefore, we conclude the results are robust to the choice of $n$, and we take $n=30$ in the following calculation. Figure 3 a shows $\Delta T$ profiles with different $\beta$ values at different surface temperatures: $\Delta T$ decreases with $\beta$ and increases with $T_{s}$. At 300-K surface temperature, the maximum of $\Delta T$ is about $0.45 \mathrm{~K}$ when $\beta=0.8$; the maximum of $\Delta T$ is about $1.1 \mathrm{~K}$ when $\beta=0.5$; and the maximum of $\Delta T$ is about $1.7 \mathrm{~K}$ when $\beta=0.2$. We find that $\Delta T$ increases faster with $T_{s}$ in drier columns, which would be used to explain the sensitivity of $\Delta$ OLR to $T_{s}$.

Equations (6)-(10) and (16) form the complete set of this model. With proper parameter values, we can estimate the magnitude of $\triangle \mathrm{OLR}$ and its change with surface temperature.

\section{f. Results}

Our calculation shows that the vapor buoyancy effect can significantly impact Earth's energy balance and future climate changes. Figure $4 \mathrm{a}$ shows that $\triangle \mathrm{OLR}$ is $O\left(4 \mathrm{~W} \mathrm{~m}^{-2}\right)$ for a wide range of parameter values. In the reference climate $\left(T_{s}=300 \mathrm{~K}\right), \Delta \mathrm{OLR}$ is about $2.5 \mathrm{~W} \mathrm{~m}^{-2}$ with $\beta=0.5$, a similar magnitude to the radiative effect due to doubling $\mathrm{CO}_{2}$. For small values of $\Delta T$, as in the present climate, the magnitude of $\beta$ is comparable to that of $\mathrm{RH}$. Therefore, a value of $\beta=0.5$ corresponds to a tropical average, while smaller values of $\beta$ might reflect subtropical dry regions [e.g., Fig. 18.3 in Vallis (2017)]. We then understand the sensitivity of $\Delta$ OLR to $T_{s}$ and $\beta$ according to Eq. (6):

1) The $\triangle$ OLR increases with $T_{s}$ at given $\beta$. This is mainly because $\Delta T$ increase with warming, as will be quantified in Figs. $4 \mathrm{~b}$ and $4 \mathrm{c}$.

2) The $\triangle$ OLR is small at both moist and dry limits. In the moist limit $(\beta \rightarrow 1), \Delta T$ is small according to Eq. (15). In the dry limit $(\beta \rightarrow 0)$, although $\Delta T$ maximizes, $\triangle \mathrm{OLR}$ is dominated by surface emission, insensitive to $\Delta T$. The OLR difference, therefore, peaks at intermediate $\beta$ values.

3) The $\Delta$ OLR peak shifts toward smaller $\beta$ in warmer climates. This is because, at high temperatures, $\Delta T$ increases faster with warming in the small- $\beta$ columns (Fig. 3a) and also because the large- $\beta$ columns become increasingly opaque to IR emission (Figs. 3b,c).

Consistent with our hypothesis, $\triangle$ OLR increases with $T_{s}$, showing a negative climate feedback. To quantify the feedback strength, we define feedback parameters

$$
\begin{gathered}
\lambda_{t}=\frac{d \Delta \mathrm{OLR}}{d T_{s}} \quad \text { and } \\
\lambda_{\mathrm{vb}}=\left.\frac{d \Delta \mathrm{OLR}}{d T_{s}}\right|_{T, \tau},
\end{gathered}
$$

where $\lambda_{t}$ is the total sensitivity of $\Delta$ OLR to $T_{s}$, and $\lambda_{\mathrm{vb}}$ is the vapor-buoyancy feedback parameter, which 

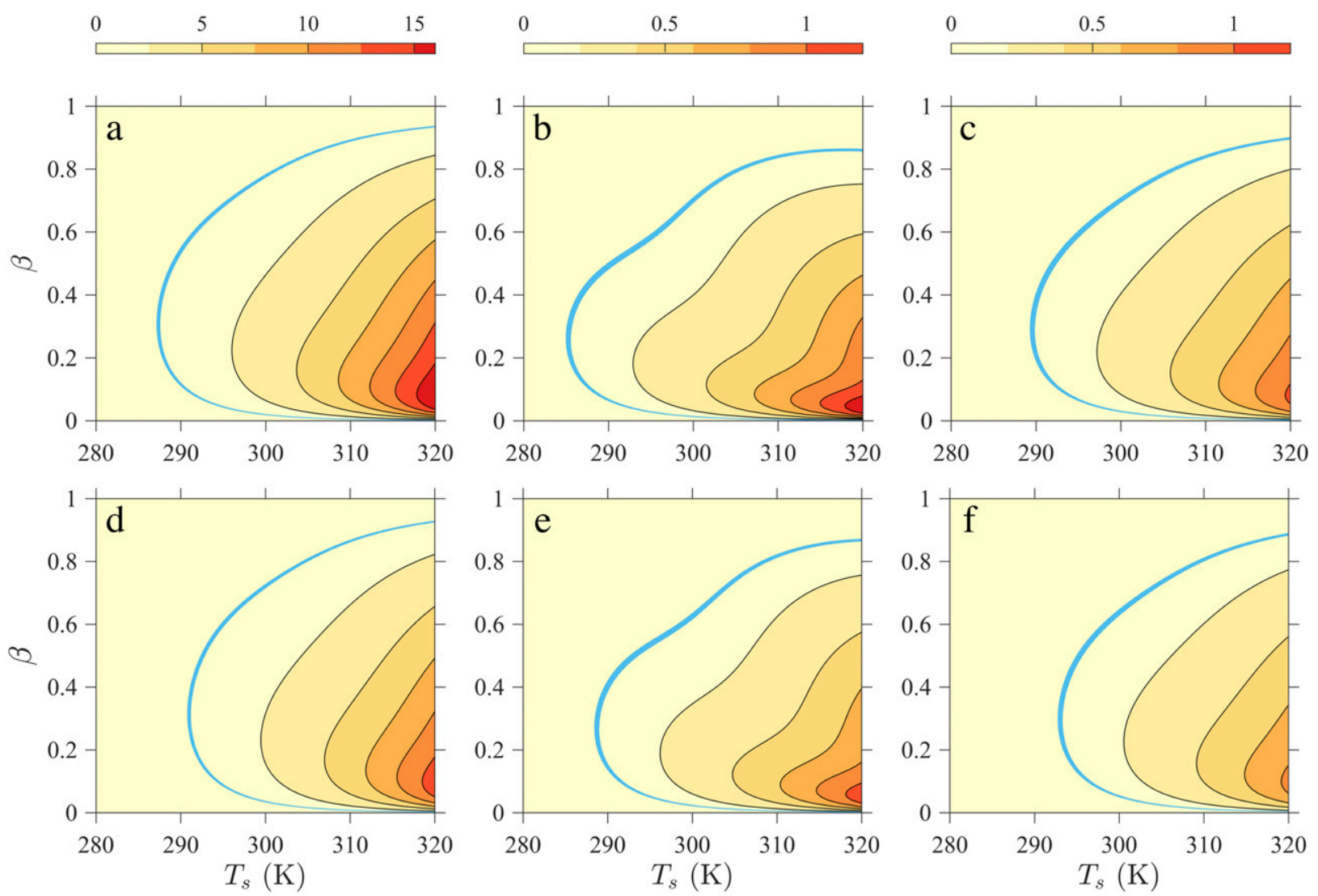

FIG. 4. (a) The full-troposphere radiative effect $\Delta \mathrm{OLR}\left(\mathrm{W} \mathrm{m}^{-2}\right)$ due to the vapor buoyancy effect. The blue curve corresponds to $\Delta \mathrm{OLR}=1 \mathrm{~W} \mathrm{~m}^{-2} ; \beta=r / r_{m}^{*}$ is a measure of dry column water vapor relative to saturated column water vapor. For small values of $\Delta T$, as in Earth's current climate, $\beta$ has a similar value to RH. (b) The total feedback parameter $\lambda_{t}\left(\mathrm{~W} \mathrm{~m}^{-2} \mathrm{~K}^{-1}\right)$ for the full troposphere. (c) The feedback parameter $\lambda_{\mathrm{vb}}\left(\mathrm{W} \mathrm{m}^{-2} \mathrm{~K}^{-1}\right)$ of the vapor buoyancy feedback for the full troposphere. The blue curve corresponds to $\lambda=0.1 \mathrm{~W} \mathrm{~m}^{-2} \mathrm{~K}^{-1}$ in (b) and (c). (d)-(f) The $\Delta$ OLR, $\lambda_{t}$, and $\lambda_{\mathrm{vb}}$ for the free troposphere $(p<900 \mathrm{hPa})$, respectively. Note that this is a negative feedback. For example, $0.1 \mathrm{~W} \mathrm{~m}^{-2} \mathrm{~K}^{-1}$ in this figure means a negative feedback of that magnitude.

only concerns $d \Delta T / d T_{s}$. That is, $\lambda_{t}$ represents the total feedback resulting from adding the vapor buoyancy effect to the atmosphere; $\lambda_{\mathrm{vb}}$ reflects only the changing temperature difference between the two atmospheres, excluding $\Delta T$ 's interaction with other changes in temperature and water vapor. Figure $4 \mathrm{~b}$ shows that $\lambda_{t}$ is of $O\left(0.2 \mathrm{~W} \mathrm{~m}^{-2} \mathrm{~K}^{-1}\right)$ in the reference climate, which compares with the feedback strength due to clouds and surface albedo. The feedback parameter keeps increasing with surface temperature and reaches about $1.4 \mathrm{~W} \mathrm{~m}^{-2} \mathrm{~K}^{-1}$ at $320 \mathrm{~K}$, suggesting that the vapor buoyancy effect becomes increasingly important in future climates.

Figure $4 \mathrm{c}$ shows that $\lambda_{\mathrm{vb}}$ is of similar magnitude to $\lambda_{t}$, suggesting the vapor-buoyancy feedback dominates the entire $\Delta$ OLR sensitivity to $T_{s}$. We find that $\lambda_{\mathrm{vb}}$ is small at the moist and dry limits. This is because $\Delta T \rightarrow 0$ when $\beta \rightarrow 1$ at all surface temperatures, and $\Delta$ OLR is dominated by surface emission when $\beta \rightarrow 0$ at all surface temperatures, not feeling $\Delta T$ and its changes. In addition, we find that the peak of $\lambda_{\mathrm{vb}}$ moves toward small- $\beta$ columns with warming because $\Delta T$ increases faster with warming at small $\beta$ columns (Fig. 3a), and also because large- $\beta$ columns become increasingly opaque at high temperature (Figs. 3b,c), insensitive to changes of $\Delta T$ that peaks in the lower troposphere.

The overall results do not depend on the assumed $\Delta T$ profiles in the boundary layer. Figures $4 \mathrm{~d}-\mathrm{f}$ shows $\Delta$ OLR, $\lambda_{t}$, and $\lambda_{\mathrm{vb}}$ for the free troposphere $(p<$ $900 \mathrm{hPa}$ ). The free-troposphere results almost reproduce the full-column results, with amplitudes that are $10 \%-15 \%$ weaker than the full-column calculation. This suggests that the vapor-buoyancy radiative effect and feedbacks occur primarily in the free troposphere.

To further test our hypothesis, we also use a real-gas radiative transfer model to calculate $\Delta$ OLR and $\lambda_{t}$. The results agree well with the two-band radiative transfer calculations. See the appendix for details. 

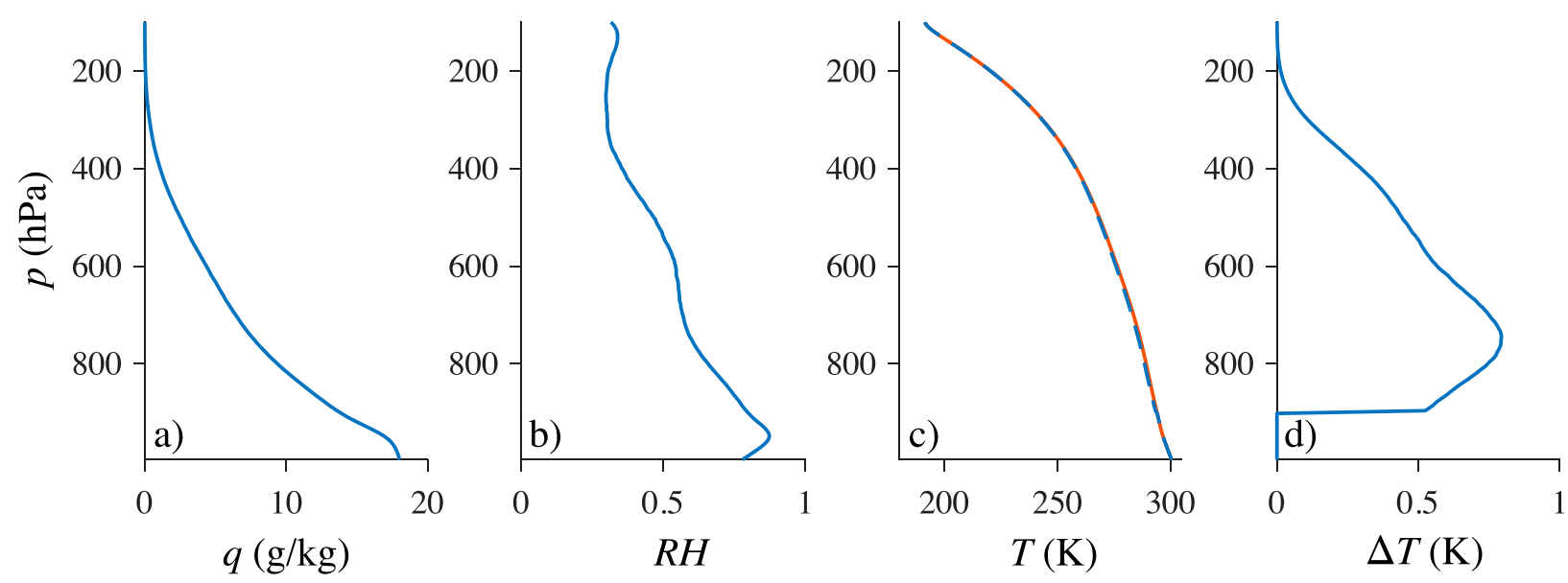

FIG. 5. Time-averaged temperature and moisture profiles at the Nauru ARM site during the period from April 2001 to August 2013: (a) specific humidity, (b) relative humidity, and (c) temperature profiles. The red curve corresponds to the observed temperature profile; the blue curve corresponds to the derived temperature profile of a convecting column. (d) Temperature difference, defined as red minus blue. Here we define the boundary layer top as $900 \mathrm{hPa}$, consistent with the definition in the simple model.

\section{Observation: A case study}

We estimate the radiative effect due to the vapor buoyancy using in situ observed temperature and moisture profiles from the Nauru Atmospheric Radiation Measurement (ARM) site during the period from April 2001 to August 2013 (Figs. 5a-c). The method of this calculation is based on the two-column model developed in Fig. 2 and in section 3. We assume that, in the free troposphere, buoyancy of this atmospheric column is the same as that of a saturated, convecting atmospheric column, and then we solve for the temperature profile of the convecting column using Eq. (13). This calculation requires $T$ (Fig. 5c) and $r$, which is based on the observed specific humidity profile (Fig. 5a). This derived temperature profile corresponds to that of an atmosphere without the vapor buoyancy effect (Fig. 2a) and is about $0.8 \mathrm{~K}$ colder than the observed temperature profile in the lower troposphere (Fig. 5d), with relative humidity about 0.6 (Fig. 5b).

We then compute the clear-sky OLR of the two columns by using the Rapid Radiative Transfer Model (RRTM v3.3; Mlawer et al. 1997). The model was run using 28 vertical levels between 1013 to $55 \mathrm{hPa}$. The $\mathrm{CO}_{2}$ mixing ratio is set at $400 \mathrm{ppm}$, and all other trace gases, including ozone, were set to zero. The clear-sky OLR calculated for the observed atmospheric column is 300.4 $\left(\mathrm{W} \mathrm{m}^{-2}\right)$; the clear-sky OLR calculated for the novapor-buoyancy column is $299.3\left(\mathrm{~W} \mathrm{~m}^{-2}\right)$. Therefore, the vapor buoyancy effect is responsible for about a $1.1 \mathrm{~W} \mathrm{~m}^{-2}$ increase in clear-sky OLR for a typical atmospheric column in the deep tropics. This result is encouraging because it agrees well with our simple model calculations at $300-\mathrm{K}$ surface temperature. If we shift the boundary layer top from 900 to $800 \mathrm{hPa}$, this OLR difference would be $0.9 \mathrm{~W} \mathrm{~m}^{-2}$, which remains significant. This sensitivity test suggests that the radiative effect of vapor buoyancy primarily originates from the free troposphere.

This case study is a first step toward quantifying the radiative effect of vapor buoyancy in Earth's atmosphere. Future analyses should expand this study by using global-scale datasets, providing a more accurate estimate of the radiative effect of vapor buoyancy over the entire tropical atmosphere.

\section{Conclusions and discussion}

The conventional wisdom is that the vapor buoyancy effect is small, so its impact on temperature is negligible in the free troposphere. However, using NASA AIRS observations, we have demonstrated that the vapor buoyancy effect could lead to about a $1.5-\mathrm{K}$ horizontal temperature difference in the lower troposphere from the driest column to the moistest column (Fig. 1), which has a significant impact on Earth's radiative balance.

Based on that novel observation, this paper proposes that the vapor buoyancy effect can increase Earth's OLR by increasing the air temperature in the dry columns. We have developed a simple model that computes the OLR difference between two atmospheres: one with the vapor buoyancy effect, and the other without this effect. We show that the magnitude of this effect is of $O\left(1 \mathrm{~W} \mathrm{~m}^{-2}\right)$ at $T_{s}=300 \mathrm{~K}$, which is then confirmed by observations, and that it increases rapidly with climate warming due to an exponential increase of atmospheric water vapor, leading to a negative climate feedback (Fig. 2b). We further show that the feedback strength 

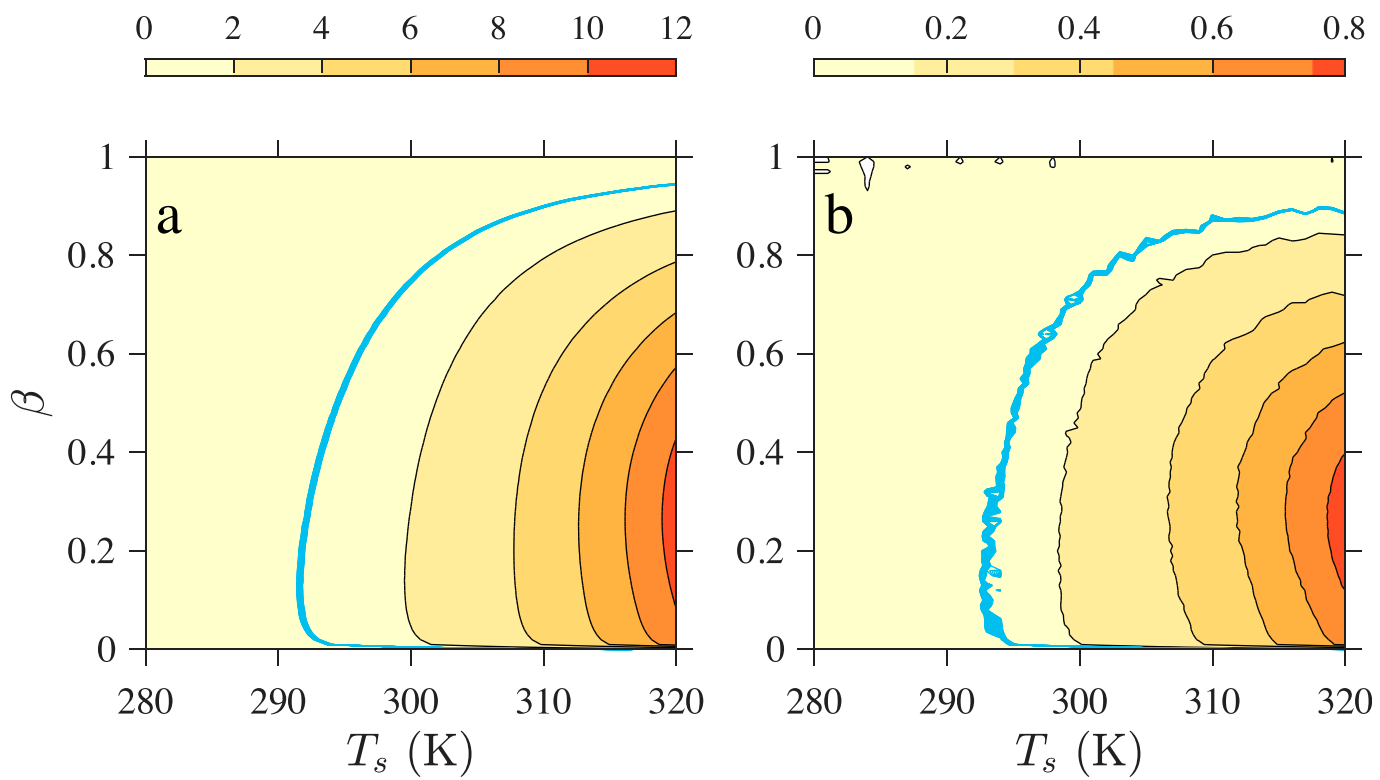

FIG. A1. RRTM results using idealized $T$ and $r$ profiles: (a) The radiative effect $\Delta$ OLR $\left(\mathrm{W} \mathrm{m}^{-2}\right)$. The blue curve corresponds to $\Delta \mathrm{OLR}=1 \mathrm{~W} \mathrm{~m}^{-2}$. (b) The total vapor-buoyancy feedback parameter $\lambda_{t}\left(\mathrm{~W} \mathrm{~m}^{-2} \mathrm{~K}^{-1}\right)$. The blue curve corresponds to $\lambda_{t}=0.1 \mathrm{~W} \mathrm{~m}^{-2} \mathrm{~K}^{-1}$.

$\lambda$ is of $O\left(0.2 \mathrm{~W} \mathrm{~m}^{-2} \mathrm{~K}^{-1}\right)$, the amplitude of which compares with major climate feedbacks, including cloud and surface albedo feedbacks. Therefore, faithful representation of the vapor buoyancy effect in climate models is necessary for accurate estimates of climate sensitivity and reliable predictions for future climate changes. While most GCMs include the vapor buoyancy effect in the model formulation, the magnitude of its associated radiative effect depends on faithfully representing the water vapor distribution in the free troposphere. In future studies, we would like to quantify this uncertainty by estimating the radiative effect of vapor buoyancy in climate models and comparing it with observations.

The vapor buoyancy effect may help explain why tropical climate has been more stable than extratropical climate (Holland and Bitz 2003; Polyakov et al. 2002; Pierrehumbert 1995). The strength of the vapor buoyancy feedback depends on water vapor contrast between moist and dry columns, which in turn depends on water vapor abundance and thereby on the temperature of the atmosphere. This effect, therefore, operates more efficiently in the tropics and less efficiently at higher latitudes. This spatial pattern may explain why fluctuations of sea surface temperature in the tropics are much smaller than that of higher latitudes in the past 100 million years (Pierrehumbert 1995).

The vapor buoyancy effect helps extend the inner edge of the habitable zone, in particular, for tidally locked exoplanets. Tidally locked planets are often slowly rotating, so their free troposphere could be in the
WBG regime globally (Koll and Abbot 2016; Mills and Abbot 2013). These planets have one fixed diurnal hemisphere and one nocturnal hemisphere, corresponding to the moist and dry columns of our model, respectively. When the tidally locked planets are approaching the inner edge of the habitable zone, their surface temperature could be significantly higher than Earth's tropical SST, providing an ideal environment for the vapor buoyancy feedback to work efficiently. However, previous studies have neglected the vapor buoyancy effect and assumed WTG (Yang et al. 2013; Yang and Abbot 2014; Pierrehumbert 2010a), which could lead to considerably narrower habitable zones. Therefore, we suggest that the vapor buoyancy effect should be accurately represented not only in GCMs but also in low-order models that are used to study climate habitability.

To focus on order-of-magnitude understanding, we have inevitably introduced simplifications to our model that only considers the clear-sky longwave radiation. An important one is that we use the two-band radiative transfer model, lacking detailed representation of water vapor's absorption spectrum. We have also assumed that $\beta$ is uniform in altitude, whereas $\beta$ often has complicated vertical structures in the real atmosphere. However, a suite of cloud-resolving model (CRM) simulations has shown similar estimates of $\triangle$ OLR and $\lambda$. The CRM uses a comprehensive radiation scheme and explicitly simulates atmospheric circulation and water vapor dynamics. The CRM results have also shown that the 
vapor buoyancy effect does not affect the shortwave radiation budget and that the clear-sky effect dominates the OLR response. The CRM results, therefore, justify our simplifications and will be presented in a companion paper (Seidel and Yang 2020).

Acknowledgments. This work was supported by Laboratory Directed Research and Development (LDRD) funding from the Lawrence Berkeley National Laboratory, provided by the Director, Office of Science, of the U.S. Department of Energy under Contract DE-AC0205CH11231, and used resources of the National Energy Research Scientific Computing Center (NERSC), also supported by the Office of Science of the U.S. Department of Energy, under Contract DE-AC02-05CH11231. This work was also supported by a Packard Fellowship for Science and Engineering awarded to author Yang. The authors thank Dr. Z. Tan and two anonymous reviewers for helpful comments and suggestions.

\section{APPENDIX}

\section{Calculation Results Using a Real-Gas Radiative Transfer Model}

We validate the simple model by using RRTM to compute $\Delta$ OLR and $\lambda_{t}$. We use the same temperature and water vapor profiles as in the simple model. To generate the model inputs, we interpolate these profiles onto 37 vertical levels from 0 to $1000 \mathrm{hPa}$. The simple model only considers a single greenhouse gas, water vapor. Therefore, we set all other trace gases, including $\mathrm{CO}_{2}$, at zero concentration.

We use RRTM to separately compute $\mathrm{OLR}_{v}$ and $\mathrm{OLR}_{\mathrm{nv}}$. We then estimate the radiative effect of vapor buoyancy as $\Delta \mathrm{OLR}=\mathrm{OLR}_{v}-\mathrm{OLR}_{\mathrm{nv}}$. From there we calculate $\lambda_{t}$ according to Eq. (17). These RRTM results (Fig. A1) agree closely with the two-band calculation of the simple model in Fig. 4. This suggests that our estimates of $\Delta$ OLR and $\lambda$ are robust.

\section{REFERENCES}

Beucler, T., and T. W. Cronin, 2016: Moisture-radiative cooling instability. J. Adv. Model. Earth Syst., 8, 1620-1640, https:// doi.org/10.1002/2016MS000763.

Bretherton, C. S., and P. K. Smolarkiewicz, 1989: Gravity waves, compensating subsidence and detrainment around cumulus clouds. J. Atmos. Sci., 46, 740-759, https://doi.org/10.1175/ 1520-0469(1989)046<0740:GWCSAD>2.0.CO;2.

_ P. N. Blossey, and M. Khairoutdinov, 2005: An energy-balance analysis of deep convective self-aggregation above uniform SST. J. Atmos. Sci., 62, 4273-4292, https://doi.org/10.1175/JAS3614.1.

Charney, J. G., 1963: A note on large-scale motions in the tropics. J. Atmos. Sci., 20, 607-609, https://doi.org/10.1175/15200469(1963)020<0607:ANOLSM>2.0.CO;2.
Collins, M., and Coauthors, 2013: Long-term climate change: Projections, commitments and irreversibility. Climate Change 2013: The Physical Science Basis, T. F. Stocker et al., Eds., Cambridge University Press, 1029-1136.

Emanuel, K. A., 1994: Atmospheric Convection. Oxford University Press, $580 \mathrm{pp}$.

Flato, G., and Coauthors, 2013: Evaluation of climate models. Climate Change 2013: The Physical Science Basis, T. F. Stocker et al., Eds., Cambridge University Press, 741-866.

Held, I. M., and B. J. Soden, 2000: Water vapor feedback and global warming. Annu. Rev. Energy Environ., 25, 441-475, https:// doi.org/10.1146/annurev.energy.25.1.441.

Holland, M. M., and C. M. Bitz, 2003: Polar amplification of climate change in coupled models. Climate Dyn., 21, 221-232, https:// doi.org/10.1007/s00382-003-0332-6.

Ingersoll, A. P., 1969: The runaway greenhouse: A history of water on Venus. J. Atmos. Sci., 26, 1191-1198, https://doi.org/ 10.1175/1520-0469(1969)026<1191:TRGAHO >2.0.CO;2.

Koll, D. D. B., and D. S. Abbot, 2016: Temperature structure and atmospheric circulation of dry tidally locked rocky exoplanets. Astrophys. J., 825, 99, https://doi.org/10.3847/0004-637X/825/2/99.

Manabe, S., and R. T. Wetherald, 1967: Thermal equilibrium of the atmosphere with a given distribution of relative humidity. J. Atmos. Sci., 24, 241-259, https://doi.org/10.1175/15200469(1967)024<0241:TEOTAW > 2.0.CO;2.

-, and - 1975 : The effects of doubling the $\mathrm{CO}_{2}$ concentration on the climate of a general circulation model. J. Atmos. Sci., 32, 3-15, https://doi.org/10.1175/1520-0469(1975) 032<0003:TEODTC $>2.0 . \mathrm{CO} ; 2$.

Mills, S. M., and D. S. Abbot, 2013: Utility of the weak temperature gradient approximation for Earth-like tidally locked exoplanets. Astrophys. J., 774, L17, https://doi.org/10.1088/20418205/774/2/L17.

Mlawer, E. J., S. J. Taubman, P. D. Brown, M. J. Iacono, and S. A. Clough, 1997: Radiative transfer for inhomogeneous atmospheres: RRTM, a validated correlated- $k$ model for the longwave. J. Geophys. Res., 102, 16 663-16 682, https://doi.org/ 10.1029/97JD00237.

Pierrehumbert, R. T., 1995: Thermostats, radiator fins, and the local runaway greenhouse. J. Atmos. Sci., 52, 1784-1806, https:// doi.org/10.1175/1520-0469(1995)052<1784:TRFATL>2.0.CO;2. _ 2010a: A palette of climates for Gliese 581g. Astrophys. J., 726, L8, https://doi.org/10.1088/2041-8205/726/1/L8.

_ 2010b: Principles of Planetary Climate. Cambridge University Press, $652 \mathrm{pp}$.

Polyakov, I. V., and Coauthors, 2002: Observationally based assessment of polar amplification of global warming. Geophys. Res. Lett., 29, 1878, https://doi.org/10.1029/2001GL011111.

Pritchard, M. S., and D. Yang, 2016: Response of the superparameterized Madden-Julian oscillation to extreme climate and basic state variation challenges a moisture mode view. J. Climate, 29, 4995-5008, https://doi.org/10.1175/JCLID-15-0790.1.

Seidel, S., and D. Yang, 2020: The lightness of water vapor helps to stabilize tropical climate. $S c i . A d v$., in press.

Sobel, A. H., J. Nilsson, and L. M. Polvani, 2001: The weak temperature gradient approximation and balanced tropical moisture waves. J. Atmos. Sci., 58, 3650-3665, https://doi.org/ 10.1175/1520-0469(2001)058<3650:TWTGAA > 2.0.CO;2.

Tompkins, A. M., 2001: Organization of tropical convection in low vertical wind shears: The role of water vapor. J. Atmos. Sci., 58, 529-545, https://doi.org/10.1175/1520-0469(2001) $058<0529$ :OOTCIL $>2.0 . \mathrm{CO} ; 2$. 
Vallis, G. K., 2017: Atmospheric and Oceanic Fluid Dynamics: Fundamentals and Large-Scale Circulation. 2nd ed. Cambridge University Press, 946 pp.

Yang, D., 2018a: Boundary layer diabatic processes, the virtual effect, and convective self-aggregation. J. Adv. Model. Earth Syst., 10, 2163-2176, https://doi.org/10.1029/2017MS001261.

,2018b: Boundary layer height and buoyancy determine the horizontal scale of convective self-aggregation. J. Atmos. Sci. 75, 469-478, https://doi.org/10.1175/JAS-D-17-0150.1.

, 2019: Convective heating leads to self-aggregation by generating available potential energy. Geophys. Res. Lett., 46, 10 687-10 696, https://doi.org/10.1029/2019GL083805.
— , and A. P. Ingersoll, 2013: Triggered convection, gravity waves, and the MJO: A shallow-water model. J. Atmos. Sci., 70, 2476-2486, https://doi.org/10.1175/JAS-D-12-0255.1.

- and 2014: A theory of the MJO horizontal scale. Geophys. Res. Lett., 41, 1059-1064, https://doi.org/10.1002/2013GL058542.

Yang, J., and D. S. Abbot, 2014: A low-order model of water vapor, clouds, and thermal emission for tidally locked terrestrial planets. Astrophys. J., 784, 155, https://doi.org/10.1088/0004-637X/784/2/155. N. B. Cowan, and D. S. Abbot, 2013: Stabilizing cloud feedback dramatically expands the habitable zone of tidally locked planets. Astrophys. J., 771, L45, https://doi.org/10.1088/ 2041-8205/771/2/L45. 